\title{
Mischpreisbildung
}

\section{Wie lässt sich das Regressrisiko mindern?}

\author{
Mischkalkulation bei neuen Medikamenten: Für Ärzte bleibt \\ ein Regressrisiko. Bei der geplanten Novellierung des \\ Arzneimittelmarktneuordnungsgesetzes wird das Problem \\ nicht gelöst. Gibt es dennoch einen Ausweg?
}

m Abschlussbericht des Pharmadialogs wird die besondere Qualität des AMNOG(Arzneimittelmarktneuordnungsgesetz)-Bewertungsverfahrens für den schnellen Zugang von Arzneiinnovationen in Deutschland herausgestellt. Ein Reformbedarf wird deswegen nur bei wenigen festgestellten Einzelproblemen gesehen. Eines von Seiten der Ärzteschaft als gravierend angesehenes Problem wird jedoch nicht angesprochen: die Mischpreisbildung bei Arzneiinnovationen mit mehreren Subgruppen, von denen ein Zusatznutzen nur in einer oder einzelnen dieser Subgruppen anerkannt worden ist.

\section{Regressanträge wegen Unwirtschaftlichkeit}

Durch die Mischkalkulation für den Erstattungsbetrag erhält eine Arzneiinnovation gegenüber dem Preis der Vergleichstherapie auch für eine Subgruppe ohne anerkannten Zusatznutzen einen höheren Erstattungspreis zuerkannt. Das veranlasst Krankenkassen leider zu gezielten Regressanträgen auch bei einer indikationsgerechten Verordnung, wobei die Ablehnung eines Zusatznutzens den Nutzen rechtlich nicht infrage stellt und der Regressantrag deswegen nur auf Unwirtschaftlichkeit gestützt werden kann. Die Begründung zum AMNOG hatte demgegenüber herausgestellt, dass es nicht mehr erforderlich sei, dass die Ärzte die Verantwortung für die Preisgünstigkeit der Arzneimittel übernehmen, weil die Verantwortung für Preise und Morbiditätsentwicklung auf die
Krankenkassen übergehen würden.

\section{AMNOG II bietet keine Lösung}

Die Verantwortung des Arztes soll danach auf die Indikationsgerechtigkeit und die Menge begrenzt, und das System der Wirtschaftlichkeitsprüfung weg von Richtgrößen auf Leitsubstanzen und Dosierungen umgestellt werden. Ein Regress wegen Unwirtschaftlichkeit einer medizinisch indizierten, aber als Folge der Mischkalkulation überteuerten Verordnung widerspricht dieser Erwartung. Wie es aktuell aussieht, wird das auf der Grundlage des Pharmadialogs erstellte AMNOG II zu diesem Widerspruch aber keine Lösung anbieten. Der vorgelegte Referentenentwurf enthält dazu keine Regelung. Eine Lösung, die entsprechend der Zielsetzung des AMNOG Arzneimittelinnovationen nach ihrem belegten Zusatznutzen vergüten soll, müsste den Erstattungspreis des Arzneimittels auf diejenigen Subgruppen ausrichten, für die ein Zusatznutzen auch tatsächlich festgestellt worden ist.

\section{Fokussierung auf Gruppen mit Zusatznutzen}

Bei der Vereinbarung oder schiedsamtlichen Festlegung des Erstattungspreises wären dann nur diejenigen Patientengruppen oder Indikationen zu berücksichtigen, bei denen Anhaltspunkte, Hinweise oder Belege für einen geringen, erheblichen oder beträchtlichen Zusatznutzen festgestellt wurden. Der Erstat- tungspreis wäre deswegen wahrscheinlich höher als der im Wege einer Mischkalkulation für die Behandlung aller in die Bewertung des Arzneimittels einbezogenen Patienten vereinbarte oder festgelegte Preis. Auf dieser Grundlage wäre eine analoge Regelung zu der bestehenden Ausnahmeregelung zur Festbetragszuordnung für nachgewiesene therapeutische Verbesserungen zu treffen.

\section{Gesetzliche Ausnahmeregelung}

Insoweit werden gemäß Paragraf $35 \mathrm{Ab}$ satz 1b Satz 9 SGB V Verordnungen für Subgruppen eines aus dem Festbetrag ausgegliederten und damit höherpreisigen Arzneimittels, für die keine therapeutische Verbesserung festgestellt wurden, ausdrücklich als unwirtschaftlich angesehen, weil hierfür die im Festbetrag verbleibenden Arzneimittel zur Verfügung stehen. Dies wären für Subgruppen von Arzneiinnovationen, für die der Gemeinsame Bundesausschuss keinen Zusatznutzen festgestellt hat, dann durch eine gesetzliche Ausnahmeregelung und eine entsprechende Preisbildung legitimiert, die Arzneimittel der Vergleichstherapie.

Dr. Rainer Hess 